\title{
IMPACTO DE LA ERRADICACIÓN DEL GATO (FELIS CATUS), EN DOS ROEDORES ENDÉMICOS DE LA ISLA CORONADOS, GOLFO DE CALIFORNIA, MÉXICO
}

\author{
Angel Rodríguez-Moreno ${ }^{1}$, Gustavo Arnaud ${ }^{1}$ y Bernie Tershy ${ }^{2}$ \\ ${ }^{1}$ Centro de Investigaciones Biológicas del Noroeste. \\ Mar Bermejo 195, Col. Playa Palo Santa Rita, La Paz, B.C.S. CP 23090, MÉXICO \\ arodri04@cibnor.mx, garnaud04@cibnor.mx \\ 2 University of California, Santa Cruz, California U.S.A.
}

RESUMEN

\begin{abstract}
Se desconoce el año en que fue introducido el gato (Felis catus) a la Isla Coronados, así como el número máximo que alcanzó su población, sin embargo, su efecto sobre los roedores nativos fue evidente, ya que los integró a su dieta, constituyendo el $70 \%$ de la misma, poniendo en peligro su supervivencia. El programa de erradicación de gatos fue iniciado en noviembre de 1998 hasta la remoción de todos los individuos en marzo del 1999. Se llevó a cabo el monitoreo de las poblaciones de Peromyscus pseudocrinitus y Chaetodipus spinatus pullus antes, durante y después del programa de erradicación, concluyendo el monitoreo en el año 2005, con el fin de identificar la eventual respuesta de los roedores a la ausencia de gatos. A corto plazo se presentó un incremento en las poblaciones de roedores, sin embargo, a largo plazo, estas fluctuaron de acuerdo a la disponibilidad de alimento y a las condiciones climáticas prevalecientes. A pesar de que no hubo evidencia numérica en las poblaciones de roedores, del beneficio de la erradicación de gatos, es claro que su remoción fue positiva, ya que su presencia representaba un riesgo potencial para las especies presentes, las cuales podrían ser extinguidas durante períodos críticos si los gatos continuaran presentes en la isla.

Palabras Clave: Peromyscus pseudocrinitus, Chaetodipus spinatus pullus, gatos asilvestrados, Isla Coronados, erradicación de gatos, Golfo de California.
\end{abstract}

\section{ABSTRACT}

It is not known when the domestic cat (Felis catus) was introduced to Coronados Island or when they reached it. Their impact on endemic rodents has been evident, rodents constitute $70 \%$ of their diet and they are putting in danger the survival of rodents. Cat eradication was initiated in November 1998 and finished in March 1999. In order to identify the eventual response of rodents to the absence of cats, a monitoring program of two resident rodents Peromyscus pseudocrinitus and Chaetodipus spinatus pullus, was undertaken before, during, and after the eradication program. The monitoring program finished in 2005. Initially an increase in rodent populations occurred followed by fluctuations in the population. Despite the fact that there was no evident benefit derived from the eradication program, it is clear that their removal was positive, since their presence represented a potential risk to endemic rodent species on the island, which could be exterminated during critical periods if feral cat continued in the island.

Key Words: Peromyscus pseudocrinitus, Chaetodipus spinatus pullus, feral cats, Coronados Island, cat eradication program, Gulf of California. 
Rodríguez-Moreno et. al.: Impacto de la erradicación del gato en dos roedores

\section{INTRODUCCIÓN}

En el Golfo de California existen 91 islas con un alto nivel de endemismos (Hall 1981, Alvarez-Castañeda \& Patton 2000, Case 2002, Lawlor et al. 2002), de las cuales numerosas especies se encuentran incluidas en la Norma Oficial Mexicana059-SEMARNAT-2001 (Diario Oficial de la Federación, 2001). Estas áreas, de gran belleza paisajística, son visitadas por turistas y pescadores, quienes establecen campamentos pesqueros provisionales en ellas, siendo de esta manera, la vía de acceso de especies exóticas. Como resultado de lo anterior, un gran número de islas en el Golfo presentan una o dos especies introducidas (López-Forment et al. 1996, Rodríguez-Moreno 1997), siendo el gato doméstico (Felis catus) el que ha tenido el mayor impacto, ya que es un depredador que ha causado importantes disminuciones en las poblaciones de fauna residente en islas del mundo, llegando incluso, a extinguirlas (Van Arde 1983, 1986, Moors \& Atkinson 1984, Brockie et al. 1988, Ebenhard 1988, Richman et al. 1988, Schofield 1989, Rodríguez et al. 1991, Arnaud et al. 1993, 1994, Alcover \& McMinn 1994), sobre todo en aquellas en las cuales las especies nativas presentan bajos números poblacionales (Smith \& Quin 1996); en contraparte, su erradicación ha propiciado la recuperación de las poblaciones de las especies residentes (Veitch 1985).

A pesar de los numerosos endemismos de fauna que se presentan en las islas del Golfo de California, existe un gran desconocimiento de sus estados de conservación. Tal es el caso de las poblaciones de pequeños mamíferos residentes de Isla Coronados, donde la rata (Neotoma bunkeri) se considera extinta (Smith et al. 1993), mientras que las poblaciones de Peromyscus pseudocrinitus y Chaetodipus spinatus pullus se encuentran en bajas densidades temiéndose que desaparezcan (Smith et al.1993, Arnaud y Acevedo 1996, Alvarez-Castañeda 1998). En este contexto, el Centro de Investigaciones Biológicas del Noroeste (CIBNOR) inició en 1998 un programa de erradicación de gatos en Isla Coronados, concluyendo en 1999 (Arnaud et al. 2000), con el fin de contribuir a la supervivencia de las especies residentes.

Sobre la erradicación y control de las poblaciones de gatos asilvestrados se ha publicado un buen número de trabajos (Veitch 1980, 1983, 1985, 1989, Veitch \& Bell 1990, Howell 1984, Rauzon 1985, Blomer \& Bester 1992, Bomford \& O’Brien, 1995, Mahlow \& Slater 1996), sin embargo, poco ha sido documentado sobre la recuperación de las poblaciones de fauna nativa de islas (Nogales et al. 2004). La escueta literatura se centra principalmente en la recuperación de aves marinas (Keitt et al. 2002, Keitt \& Tershy 2003, Cooper et al. 1995), no quedando claros los beneficios de la conservación para otros grupos faunísticos, como resultado de los programas de erradicación. En este contexto, este sería un estudio pionero en documentar la respuesta de la población de dos especies de roedores de una isla, al menos, en islas mexicanas. 


\section{MATERIAL Y MÉTODOS}

Área de Estudio: Isla Coronados, localizada a $2.6 \mathrm{~km}$ al este de la península de Baja

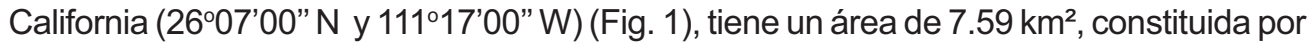
un volcán de $283 \mathrm{~m}$ de altura y planicies arenosas (Murphy \& Aguirre-León 2002). La precipitación y temperaturas promedio, basadas en 20 años de observaciones, de acuerdo a la estación climatológica de la ciudad de Loreto, es de $155.7 \mathrm{~mm}$, con $24.8^{\circ} \mathrm{C}$ (mínima de $17.6^{\circ} \mathrm{C}$ y máxima de $31.7^{\circ} \mathrm{C}$ ). Las lluvias se presentan en dos períodos, de julio a octubre (lluvias de verano) y de noviembre a febrero (lluvias de invierno), siendo septiembre el mes mas lluvioso; durante los meses de marzo a junio se presenta el período de sequía (García, 1980). Fisionómicamente la isla presenta seis tipos de hábitat: 1) Áreas costeras: donde se incluyen playas y dunas con vegetación establecida; 2) Planicies halófilas; 3 ) Planicies de Matorral xerófilo; 4) Cañadas; 5) Áreas rocosas, con piedras de un diámetro mayor $30 \mathrm{~cm}$, y 6) Áreas pedregosas, con piedras de diámetro menor a los $30 \mathrm{~cm}$. Las plantas dominantes en la isla incluyen: matacora (Jatropha cuneata), palo adán (Fouquiera digetii), torote (Bursera microphylla), pitahaya agria (Stenocereus gummosus), mangle dulce (Maytenus phyllantoides). La fauna está constituida por tres especies de roedores (Hall, 1981) de los cuales una se considera extinta. (Smith et al. 1993), por 15 especies de reptiles (Grismer 2002) y por 16 especies de aves terrestres (Case et al. 2002) En la isla no existe agua dulce, ni está habitada.

Trampeo de gatos: Para la captura de gatos se utilizaron trampas, de las cuales 106 fueron trampas de jaula (tomahawk $81 \times 25.5 \times 30.5 \mathrm{~cm}$ ), 215 trampas de doble muelle (cepos) y 67 lazos para captura con nudo corredizo (snares) $(n=388)$, distribuidas en seis periodos de tiempo, de noviembre de 1998 a marzo de 1999. Las trampas fueron colocadas dependiendo de la presencia de huellas o excretas de gatos en un área y situadas estratégicamente en pasajes entre rocas o vegetación. Los métodos fueron similares a los descritos por Veitch (1985). En las zonas rocosas y pedregosas fueron colocadas las trampas de jaula (cebadas con sardina) y los lazos de nudo corredizo, ante la imposibilidad de utilizar aquí los cepos; mientras que en las planicies arenosas, cada trampa de jaula fue revisada diariamente. Al concluir cada estancia y antes de partir de la isla, se dejó colocada una red de trampas de doble muelle y lazos de nudo corredizo, con el fin de que el trampeo se mantuviera en nuestra ausencia. Dada la presencia permanente de turistas en la isla, las trampas fueron colocadas en cañadas y sitios alejados de las playas turísticas, no así de los campamentos pesqueros. Los animales capturados fueron sacrificados utilizando una sobredosis de cloroformo.

Una vez que ya no se capturaron gatos, se continuó con el trampeo, utilizando además trampas de huellas, constituida por arena de grano delgado, cernida en un metro de diámetro, colocando en el centro un atrayente (pescado fresco o sardina enlatada), además de cámaras fotográficas con sensor de movimiento (Deer Cam). Se continuó con la búsqueda exhaustiva de huellas, excrementos y cualquier otro indicio de la presencia de gatos.

Presencia de roedores en excretas: Se colectaron 100 excretas de gato durante 1998, para determinar el porcentaje de ellas en las que aparecían roedores, separados 
Rodríguez-Moreno et. al.: Impacto de la erradicación del gato en dos roedores

por especie; los restos no digeridos de éstos (pelo y huesos), fueron identificados en laboratorio con la ayuda de un microscopio estereoscópico, a través de su comparación con material de referencia (ejemplares de la colección mastozoológica del CIBNOR). Los datos fueron expresados en porcentaje de ocurrencia.

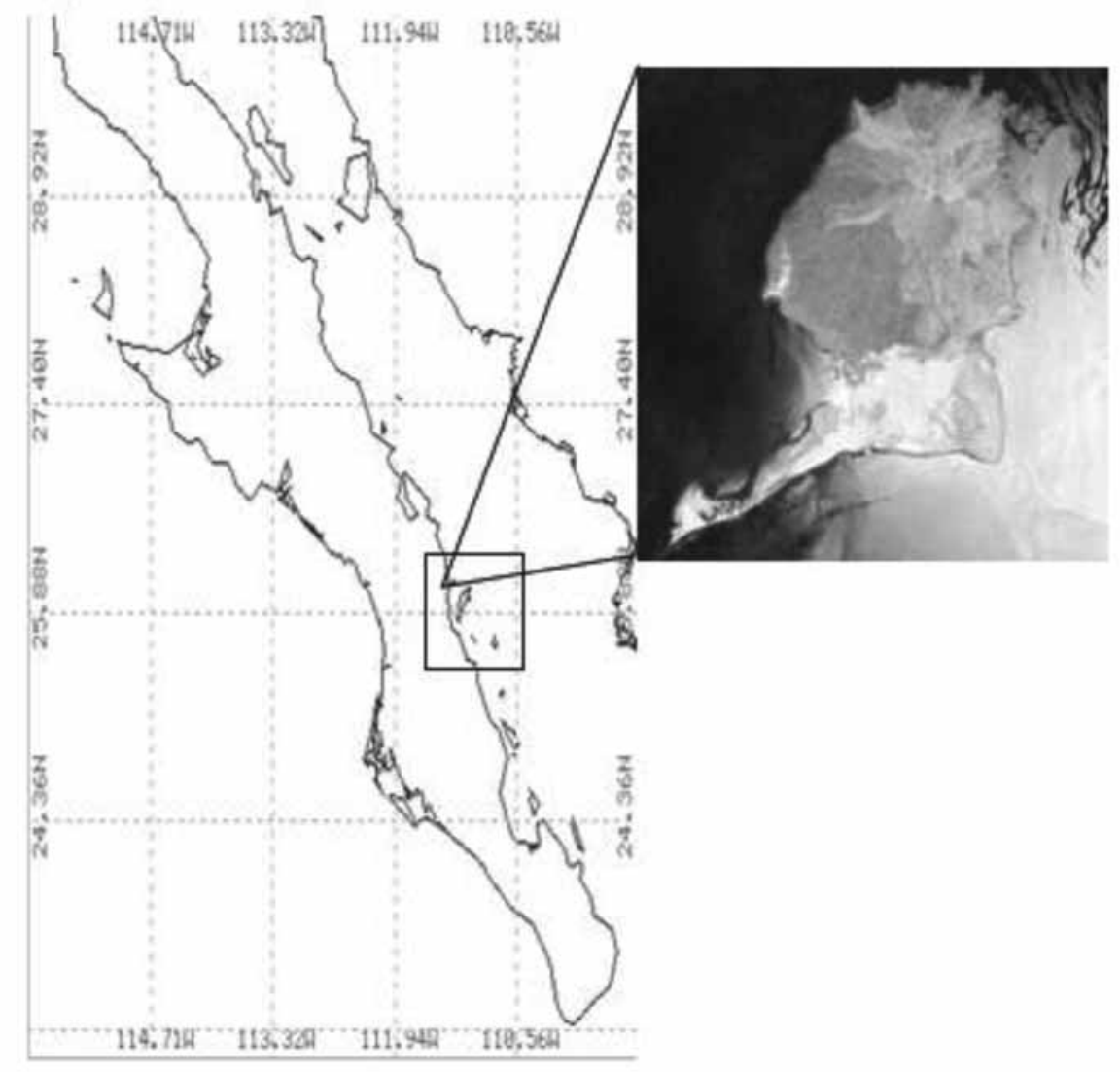

Figura 1

Localización de Isla Coronados, en el Golfo de California.

Monitoreo de roedores: El monitoreo se llevó a cabo durante tres noches consecutivas en 14 muestreos, de junio 1998 a septiembre 2002 (jun, oct 98, ene, may, jul, sept, dic 1999; feb, sept 2000; abr, jul, oct 2001; feb, sept, 2002), en los hábitat rocosos y planicies de matorral; siete muestreos se llevaron a cabo en las planicies halófilas (oct 1998 - feb 2000). Solo en el hábitat de planicies de matorral se continuaron los muestreos de septiembre del 2002 a junio del 2005, llevando a cabo nueve de ellos. Se utilizaron 
trampas Sherman de aluminio $(75 \times 90 \times 230 \mathrm{~mm})$, cebadas con hojuelas de avena puestas al atardecer y revisadas al amanecer. El trampeo en la planicie de matorral xerófilo se llevó a cabo en una hectárea, donde se colocaron 6 líneas de 11 trampas con una separación de 10 metros entre cada una y de 20 metros entre las líneas ( $n=$ 66 trampas); en el hábitat rocoso, igualmente se cubrió una superficie de una hectárea de trampeo ( $n=60$ trampas), mientras que en las planicies halófilas, se cubrió una superficie de 0.48 ha de trampeo, donde se colocaron dos líneas de 15 trampas cada una, con una separación de 10 metros entre ellas y de 20 metros entre las líneas $(n=30)$. De los animales capturados se registró: especie, sexo, peso, clase de edad y condición reproductiva, posteriormente se marcaron a través de ecotomización de falanges, siendo liberados en el mismo sitio de captura. Se estimó la densidad por especie y por hábitat utilizando el Método de Petersen de captura y recaptura (W.W.F. 1980, White et al. 1982, Anderson et al. 1983). Los resultados obtenidos se correlacionaron con el programa de erradicación de Felis catus. La existencia de diferencias significativas entre las medias mensuales se estimó aplicando la prueba de análisis de varianza de una vía con dos factores, siguiendo el procedimiento estadístico de Studen-Newman-Keuls (Sokal \& Rohlf 1981).

La distribución de los roedores en la isla fue determinada a través de su trampeo en los diferentes hábitat, tanto del norte, sur, este y oeste. Se utilizaron 4,321 trampas/noche. Los datos obtenidos fueron expresados en abundancias tales como: común (cuando eran capturados en más de 1/3 de las trampas utilizadas por sitio), escaso (cuando ocurrieron en menos de $1 / 3$ de las trampas) y ausente (cuando no fue capturado ningún individuo).

\section{RESULTADOS}

Presencia de roedores en excretas: Fueron encontrados restos no digeridos de roedores en el $70 \%$ de las excretas de gatos, de ellos, el $68 \%$ correspondió a C. spinatus pullus y el $32 \%$ a $P$. pseudocrinitus.

Trampeo de gatos: El trampeo y captura de gatos se llevó a cabo de noviembre de 1998 a marzo de 1999, habiendo capturado ocho individuos adultos, de los cuales siete fueron capturados con cepos y uno con trampa de jaula. En tres de los individuos capturados se encontraron en sus estómagos ratones de la especie Ch. spinatus pullus. De marzo a julio de 1999, se continuó el trampeo usando también trampas para huellas y cámara con sensor de movimiento. No se capturó ningún individuo ni se encontraron indicios de su presencia durante este período (Cuadro 1). En dos playas de la isla, durante mayo y julio de 1999 , se vigilaron los restos de pescado destazados por los pescadores y dejados en las playas con la finalidad de detectar las huellas de los gatos, ya que en el pasado éstos se acercaban a los campamentos pesqueros para alimentarse de dichos restos, sin embargo ningún gato se acercó a ellos.

Distribución de roedores: Chaetodipus spinatus pullus se presentó en todos los hábitats de la isla, siendo común en las planicies de matorral xerófilo y cañadas; en las áreas rocosas y pedregosas fue escaso, estando asociado a los manchones de vegetación; 
Rodríguez-Moreno et. al.: Impacto de la erradicación del gato en dos roedores

fue igualmente escaso en las planicies halófilas y dunas. Por su parte $P$. pseudocrinitus estuvo ausente de las dunas costeras, fue escaso en las planicies de matorral y halófilas, pero común en las áreas rocosas, pedregosas y en las cañadas.

Monitoreo de roedores: La densidad de las dos especies de roedores mostraron fluctuaciones a través del período de muestreo, la cual varió de una densidad inicial para Ch. spinatus pullus en junio de 1998 (en hábitat de rocas: $17 \mathrm{ind} / \mathrm{ha}$, planicies de matorral: $50 \mathrm{ind} / \mathrm{ha}$ ); hasta alcanzar valores máximos durante septiembre de 1999, en rocas: 93 ind/ha, planicies de matorral: 85 ind/ha, para posteriormente disminuir paulatinamente la densidad de Ch. spinatus pullus en los dos hábitats, hasta alcanzar valores mínimos en octubre de 2001 (rocas: 16 ind/ha, planicies de matorral: 24 ind/ha); Los valores de densidad se incrementaron posteriormente hasta septiembre 2002 (en rocas: 75 ind/ha, planicies de matorral: 52 ind/ha); De abril de 2003 a junio de 2005 en la planicie de matorral, Ch. spinatus pullus presentó incrementos y descensos en su valor de población, variando entre un rango de 44-108 ind/ha, con el valor de densidad máximo durante junio 2005 y el mínimo durante marzo 2005 (Fig. 3).

Mientras que para P. pseudocrinitus la densidad inicial en junio de 1998 (en el hábitat de rocas: $19 \mathrm{ind} / \mathrm{ha}$ ), incrementándose hasta alcanzar valores máximos durante septiembre de 1999 (P. pseudocrinitus en rocas: 51 ind/ha), para posteriormente presentar una abrupta disminución en su densidad durante diciembre 2000 y febrero 2001 (13 ind/ ha en cada mes), con un posterior incremento durante los meses de septiembre 2000 , abril y julio 2001 (35 ind/ha), disminuyendo, al igual que las poblaciones de Ch. spinatus pullus, durante el mes de octubre 2001. Los valores de densidad se incrementaron posteriormente hasta septiembre 2002 P. pseudocrinitus (en rocas: 64 ind/ha) (Fig. 2). En general, $C h$. spinatus pullus presentó mayores densidades que las de $P$. pseudocrinitus en el hábitat rocoso. En las planicies halófilas, la captura de roedores fue escasa e irregular, $C h$. spinatus pullus presentó un valor de densidad máximo durante septiembre 1999 (6 ind/ha), mientras que P. pseudocrinitus su mayor valor de densidad fue en diciembre de ese mismo año ( 2 ind/ha).

Correlación roedores-gatos: En relación a los cambios de las densidades de los roedores y la erradicación de los gatos, se observaron diferencias de los valores obtenidos entre el inicio de la erradicación y después de ésta. Los valores de densidad se incrementaron de tal manera que en el matorral xerófilo hubo una diferencia de $35 \mathrm{ind} / \mathrm{ha}$ para C. spinatus; mientras que en las laderas rocosas se observó en $P$. pseudocrinitus un incremento de 76 ind/ha y en C. spinatus 33 ind/ha (Fig. 4), siendo estos incrementos estadísticamente significativos $\left(X^{2}=117.32>P .05\right)$.

\section{DISCUSIÓN}

Si bien se desconoce el año en que fue introducido el gato a Isla Coronados, así como el número máximo que alcanzó su población, su efecto sobre los roedores nativos fue evidente, ya que los integró a su dieta, constituyendo un componente importante de la misma, dado que se encontraron en un $70 \%$ de las excretas analizadas, lo cual fue 


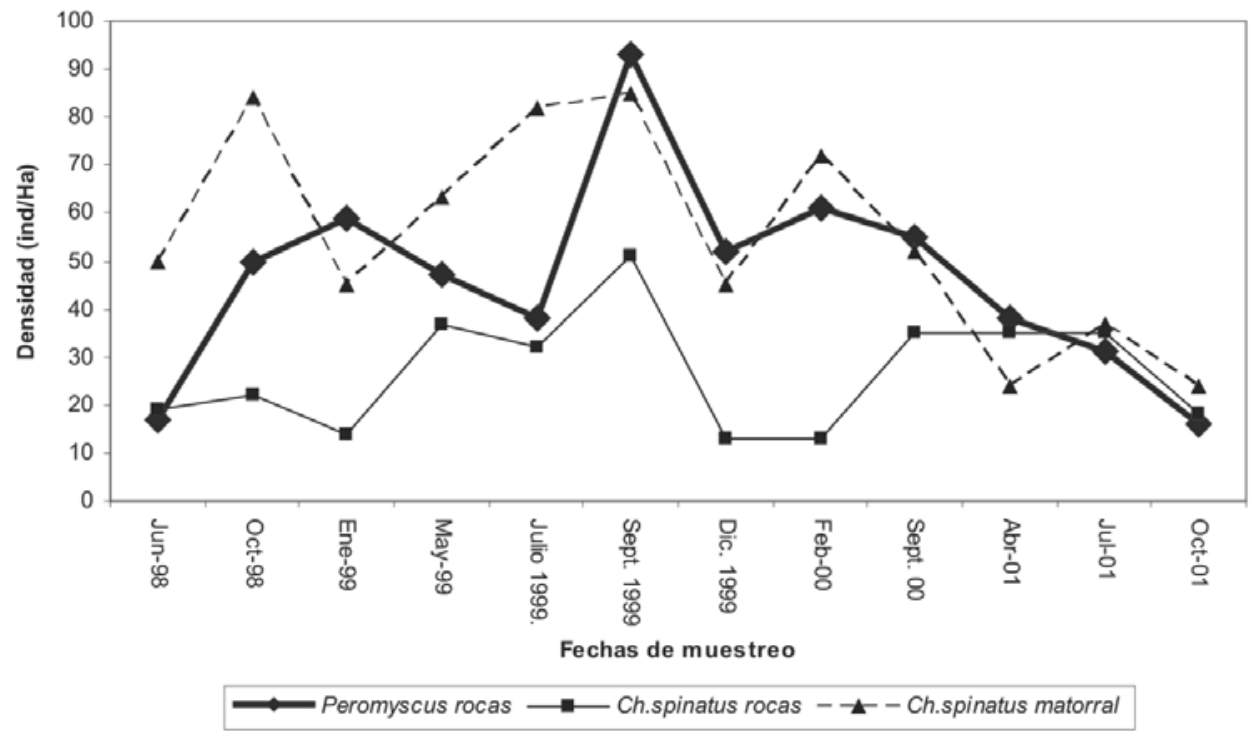

Figura 2

Densidad de Chaetodipus spinatus pullus y de Peromyscus pseudocrinitus en los hábitat rocosos y de planicie de matorral xerófilo, de 1998 a 2001.

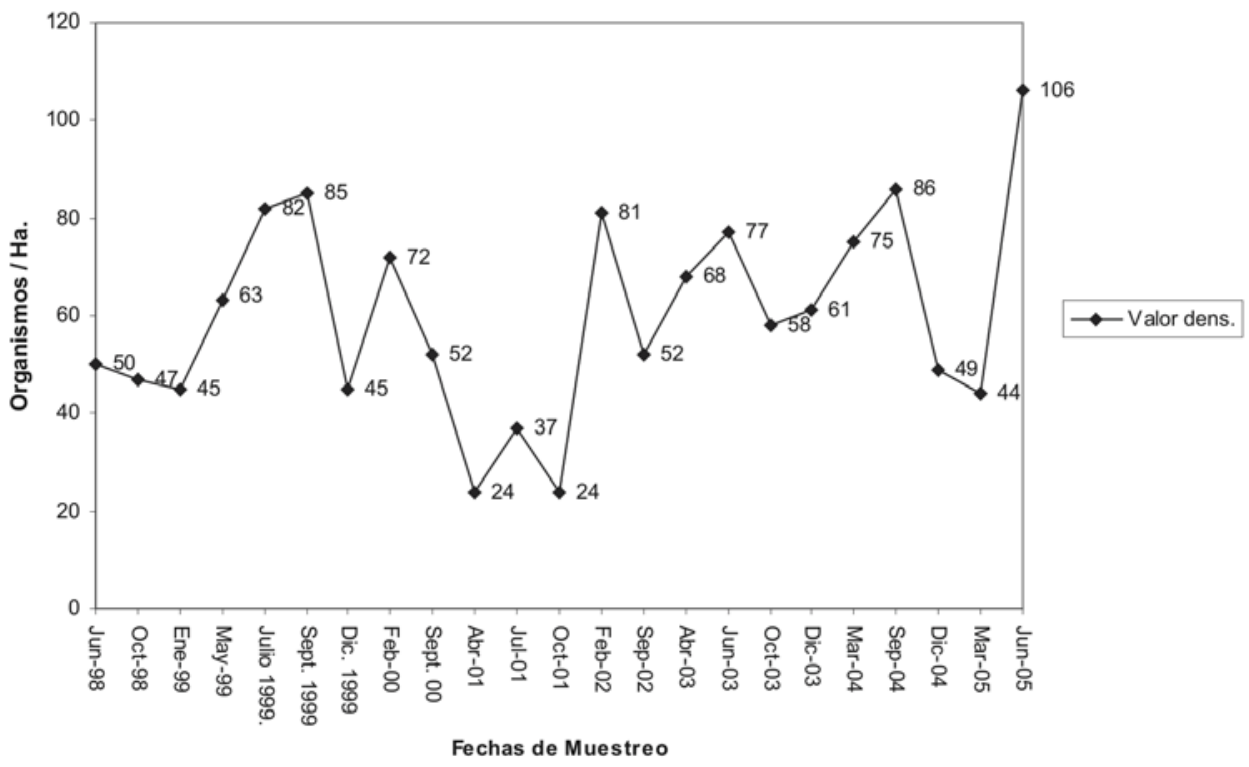

Figura 3

Densidad de Chaetodipus spinatus pullus en planicies de matorral xerófilo de junio de 1998 a junio 2005. 
Rodríguez-Moreno et. al.: Impacto de la erradicación del gato en dos roedores

similar a otras islas donde se reporta la presencia de gatos y roedores (Langham 1990, Tidemann et al. 1994, Nogales \& Medina 1996). La mayor depredación, sobre Ch. spinatus pullus (68\%), reflejó la preferencia del gato por el hábitat de planicies de matorral xerófilo para obtener alimento, debido a que en ese sitio los roedores son más vulnerables, dada la escasa cobertura vegetal; en contraste, el hábitat rocoso, que ofrece mayor posibilidad de escape a los roedores, implicó que P. pseudocrinitus fuera menos depredado (32\%), a pesar de su abundancia. La presencia significativa de excretas y huellas de estos roedores en este hábitat apoyan lo anterior.

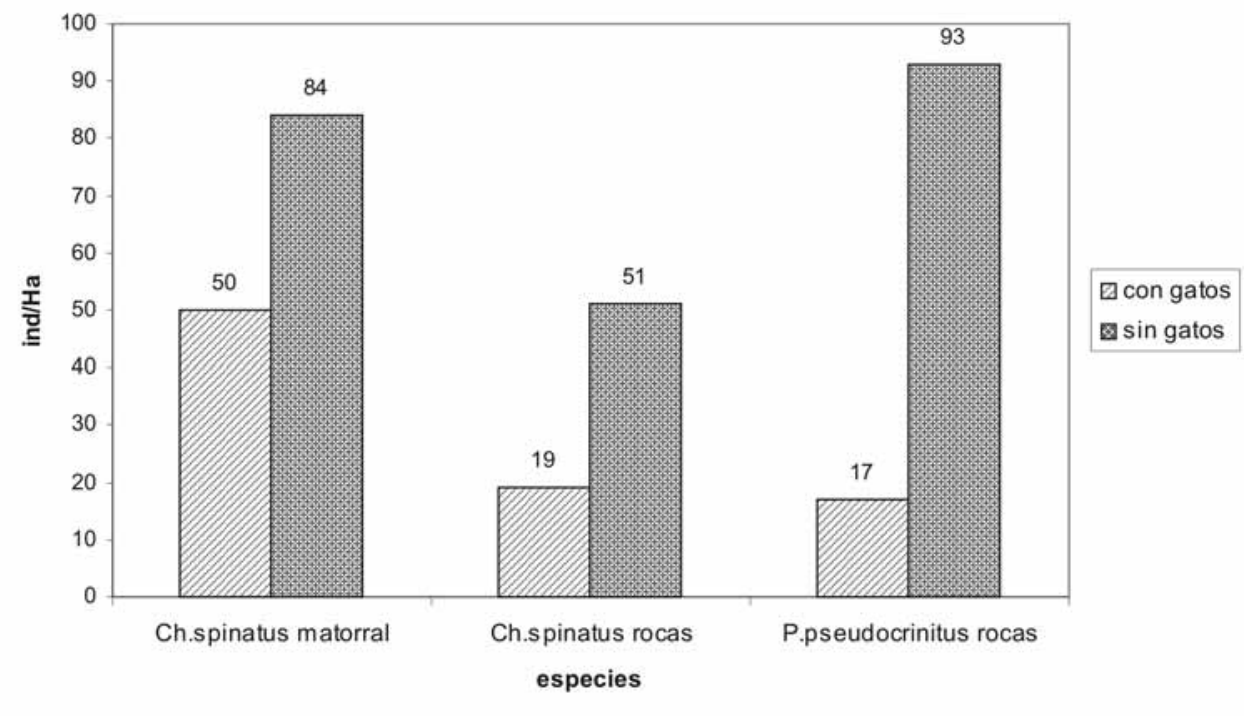

Figura 4

Diferencia en la densidad de roedores en los hábitat de rocas y de planicies con matorral xerófilo, en relación a la presencia / ausencia de gatos.

Con el monitoreo de las poblaciones de roedores, se pretendió hacer evidente la respuesta de los roedores a la erradicación de gatos. Los valores de densidad de los roedores se incrementaron de tal manera que en el matorral xerófilo hubo una diferencia de 35 ind/ha para Ch. spinatus; mientras que en los hábitat rocosos se observaron en $P$. pseudocrinitus un incremento de 76 ind/ha y en Ch. spinatus de 33 ind/ha. Si bien se reportan incrementos en las densidades de las poblaciones residentes en islas, posterior a las erradicaciones (Island Conservation \& Ecology Group 1998, Blommer \& Bester 1992, Bramley 1996, Key \& Muñoz 1994, Veitch 1985, Towns 1991), dichos estudios son muy puntuales, desarrollados en un periodo de tiempo corto, de tal forma que los resultados obtenidos pueden ser inconsistentes. Se requieren realizar monitoreos a largo plazo, con el fin de tener mayor claridad en los beneficios de la erradicación de los exóticos en relación a las especies residentes. Así mismo, es necesario considerar la disponibilidad de recursos (alimento, cobertura vegetal, calidad de hábitat) y factores medioambientales. 
Si nuestro estudio hubiese sido concluido en septiembre de 1999, se hubiera concluido, en términos numéricos, el beneficio de la erradicación de los gatos, ya que en ese momento el análisis de la población evidenció diferencias estadísticas significativas, sin embargo, dichas diferencias no pueden ser atribuidas a la erradicación del gato, ya que en muestreos posteriores (1999-2001), las poblaciones se redujeron. Ch. spinatus pullus y P. pseudocrinitus mostraron similares fluctuaciones de densidad, con picos poblacionales durante los períodos de lluvias de verano (julio-octubre) de 1999 y 2000; los valores mínimos se observaron al finalizar la época de lluvias del 2001. Dado que las dos especies consumen recursos alimenticios aparentemente similares (Jameson \& Peters 1988), es evidente que la disponibilidad de alimento es un factor que influye directamente sobre su reproducción y demografía, como ha sido mostrado en otros roedores (Millar 1988). En Baja California Sur la floración y producción de semillas presenta picos en la época de lluvias (León \& Coria 1992; León de la Luz et al. 1996), lo cual sugiere que la disponibilidad de alimento alcanza sus valores máximos durante este período del año. Las densidades obtenidas en el presente trabajo, son comparables a las reportadas para otras especies de roedores en otras regiones áridas como la península de Baja California (Gómez-Ezqueda 1996, Trujano 2000) y Sonora (GonzálezRomero 1995), lo cual indica una similitud en el comportamiento de las poblaciones de roedores, las cuales se incrementan en la temporada de lluvias. En heterómidos, se ha demostrado que las fluctuaciones de sus poblaciones están correlacionadas con la producción de frutos, semillas y plántulas, como resultado directo de la precipitación (Braun 1985), mientras que en los cricétidos, las fluctuaciones cíclicas

\section{Cuadro 1}

Períodos de trampeo, trampas utilizadas y número de gatos asilvestrados capturados durante la erradicación. * Período en el cual las trampas fueron dispuestas en la isla, estando ausente el equipo de erradicación.

\begin{tabular}{lccccc}
\hline $\begin{array}{c}\text { Períodos de trampeo } \\
\text { de gatos }\end{array}$ & $\begin{array}{c}\text { No. de } \\
\text { trampas } \\
\text { de jaula }\end{array}$ & $\begin{array}{c}\text { No. de } \\
\text { cepos }\end{array}$ & $\begin{array}{c}\text { No. Trampas } \\
\text { de nudo } \\
\text { corredizo }\end{array}$ & $\begin{array}{c}\text { No. de } \\
\text { trampas de } \\
\text { huella }\end{array}$ & $\begin{array}{c}\text { No. de gatos } \\
\text { capturados }\end{array}$ \\
\hline 11-16 Nov.98 & 50 & 40 & - & - & 1 \\
1-9 Dic.98 & 18 & 30 & 8 & - & 2 \\
10-24 Ene.99* & - & 13 & 8 & - & 3 \\
25-31 Ene.99 & 18 & 23 & 8 & - & 0 \\
1 Feb - 15Marzo 99* & - & 13 & 8 & - & 1 \\
16-21 Marzo 99 & 10 & 25 & 7 & 5 & 1 \\
22Marz.-2 May.99* & - & 13 & 7 & 3 & 0 \\
3-9 May.99 & 10 & 25 & 7 & 3 & 0 \\
10May-5Jul.99* & - & 13 & 7 & 3 & 0 \\
6-11Jul.99 & - & 20 & 7 & 2 & 0 \\
Totales & 106 & 215 & 67 & 16 & 8 \\
\hline
\end{tabular}


Rodríguez-Moreno et. al.: Impacto de la erradicación del gato en dos roedores

de sus poblaciones, presentan ciclos de bajas y altas densidades alternado cada dos años (Krebs \& Meyer 1974).

Los valores poblacionales en este estudio, contrastan con los reportados en esta misma isla durante la primera parte de la década de los noventas, los cuales fueron significativamente bajos (Smith et al.1993, Alvarez-Castañeda 1998, Arnaud \& Troyo 1995), siendo posible que las bajas capturas ocurrieran durante un período de baja densidad correspondiente a un comportamiento cíclico, lo cual es común en roedores (González-Romero 1995, Miller \& Miller 1995). Las fluctuaciones de Ch. spinatus pullus, del 2001 al 2005 apoyan que los cambios poblacionales son el resultado de la disponibilidad de alimento y las condiciones climáticas prevalecientes.

Es posible que bajo condiciones de estrés medioambiental (periodos prolongados de sequía y reducida disponibilidad de alimento, o bien, precipitaciones excesivas, como las que ocurren durante los períodos de huracanes en el Pacífico), las poblaciones de roedores disminuyan, siendo sensibles a la depredación, poniendo en riesgo su supervivencia. En este contexto, a pesar de que en el presente estudio no se mostró evidencia del beneficio de la erradicación de gatos en las poblaciones de roedores a largo plazo, es claro que su presencia representaba un riesgo potencial para las especies presentes, las cuales podrían ser extinguidas durante períodos críticos si los gatos continuaran presentes.

\section{AGRADECIMIENTOS}

Los autores agradecen al Parque Nacional Bahía de Loreto el apoyo logístico otorgado y en particular a su exdirector Benito Bermúdez, así como a su actual director Roberto López. Se agradece el financiamiento otorgado por la Comisión Nacional para el Uso y Aprovechamiento de la Biodiversidad (CONABIO) proy. Q042; por el Fondo Mexicano para la Conservación de la Naturaleza (FMCN) proy. A1-99-012 y de los fondos mixtos SEMARNATCONACyT proy. 2002-CO1-0584. Se agradece al Laboratorio de Ecología del CIBNOR, por su apoyo en el procesamiento de las excretas de gato y a Ira Fogel e Iván Arnaud por la traducción y revisión del resumen en inglés.

\section{LITERATURA CITADA}

Alcover, J. \& M. McMinn. 1994. Predators of vertebrates on islands. BioScience 44(1):12-18. Alvarez-Castañeda, S. 1998. Peromyscus pseudocrinitus. Mammalian Species No. 601 pp. 1- 3

Alvarez-Castañeda, S. \& J. P. Patton. 2000. Mamíferos del Noroeste de México. Vols: I y II. Centro de Investigaciones Biológicas del Noroeste Baja California Sur, México.

Anderson, D., Burnham, K., White, G. \& D. Otis. 1983. Density Estimation of small populations using a trapping web and distance sampling methods. Ecology 64(4):674-680.

Arnaud, G. \& M. Acevedo. 1996. Crisis de sobrevivencia de tres especies de roedores endémicos de Isla Coronados, Golfo de California, debido a la presencia de especies exóticas. In: Resúmenes III Congreso Nacional de Mastozoología. Asociación Mexicana de Mastozoología. 
Arnaud, G., A. Rodríguez \& S. Alvarez. 1994. El gato doméstico (Felis catus) implicaciones de su presencia y alternativas para su erradicación. 16: 319-328. In: Ortega y Castellanos (eds). La Isla Socorro, Reserva de la Biosfera, Archipiélago de Revillagigedo, México. Centro de Investigaciones Biológicas del Noroeste S.C.

Arnaud, G., A. Rodríguez., A. Ortega-Rubio \& S. Alvarez-Cárdenas. 1993. Predation by cats on the unique endemic lizard of Socorro Island (Urosaurus auriculatus) Revillagigedo, México. Ohio J. Sci. 93(4): 101-104.

Arnaud, G., A. Rodríguez-Moreno \& M. Camacho. 2000. Programa exitoso de erradicación de gatos en Isla Coronados, B.C.S. Insulario 11-12: 21-24.

Arnaud, G. \& E. Troyo. 1995. Populations of Peromyscus pseudocrinitus in Coronados Island, Gulf of California, México. Peromyscus Newsletter 21:20 - 21.

Bloomer, J. \& M.N., Bester. 1992. Control of feral Cats on sub-antartic Marion Island, Indian Ocean. Biological Conservation. 60: 211-219

Bomford, M. \& P. O'Brien. 1995. Eradication or control for vertebrate pests. Wildlife Soc. Bull. 23(2): $249-255$.

Bramley, G. 1996. A small predator removal experiment to protect North island Weka (Gallirallus australis greyi) and the case for single-subject approches in determing agents decline. New Zealand J. Ecol.. 20(1): 37-43.

Braun E. S. 1985. Home Range and activity patterns of the giant Kangaroo rat, Dipodomys ingens. J. Mammal. 66: 111-112.

Brockie, R.E., L.L. Loope, M.B. Usher \& O. Hamann 1988. Biological invasiones of island nature reserves. Biological Conservation 44:9-37.

Case, T. 2002. Reptiles: Ecology. 221-270 pp. In: Case, Cody \& Ezcurra (eds.). New Isaland Biogeography of the Sea Cortes. Universty of California Press.

Case T., M. L. Cody \& E. Ezcurra. 2002. A New Isaland Biogeography of the Sea Cortes. Universty of California Press. 869 pp.

Cooper J., A.V. Marais, J.P. Bloomer \& M. Bester. 1995. A success story: breeding of burrowing petrels (Procellariidae) before and after the eradication of feral cats Felis catus at subantartic Marion Island. Marine Ornithology 23:33-37.

Diario Oficial de la Federación. 2001. Norma Oficial Mexicana NOM 059-SEMARNAT.

Ebenhard, T. 1988. Introduced birds and mammals and their ecological effects. Swedish Wildlife Research. 13(4):1- 53.

García, E. 1980. Modificaciones al sistema de clasificación climática de Köppen. México. 81 págs.

Gomez-Ezqueda, L. 1996. Análisis comparativo de la densidad poblacional de roedores (Rodentia) en dos hábitats (Vegetación mesófila, Matorral xerófilo) de San Pedro de la Presa, B.C.S. Tesis de Licenciatura Universidad Nacional Autónoma de México. 49 pp.

González-Romero, A. 1995. Cambios en la composición de las comunidades de roedores con relación a los tipos de vegetación y geomorfología en el Pinacate, Sonora, México. Acta Zoológica Mexicana (n.s.) 64: 45-58.

Grismer, L.L. 2002. Amphibians and Reptiles of Baja California. University of California Press. xiii +399 pp.

Hall, R. 1981. The Mammals of North America. The Ronald Press Company. Vol.II.

Howell, P. 1984. An evaluation of the biological control of the feral cat Felis catus (Linnaeus, 1758). Acta Zool. Fennica 172:111-113.

Island Conservation \& Ecology Group. 1998. Protección de los Ecosistemas Insulares de Baja California. Reporte Técnico Final 1997-1998. 1-16 pp. 
Rodríguez-Moreno et. al.: Impacto de la erradicación del gato en dos roedores

Jameson E.W. \& H. Peters. 1988. California Mammals. University of California Press xii+403 $\mathrm{pp}$.

Keitt, B. \& B. Tershy. 2003. Cat eradication significantly reduces shearwaters mortality. Animal Conservation 6:307-308.

Keitt, B., C. Wilcox, B. Tershy, D. Croll \& C. Donland. 2002. The effect of feral cats on the population viability of Black-vented Shearwaters (Puffinus opistbomelas) on Natividad Island, México. Animal Conservation 5:217-223.

Key, G., \& E., Muñoz 1994. Distribution and current status of rodents in the Galápagos. Noticias de Galápagos 53 :21-25

Krebs, C.J. \& J.H. Meyer. 1974. Population cycles in small mammals. Advances in Ecological Research, 8:267-399.

Langham, N.P. 1990. The diet of Feral cats (Felis catus L.) on Hawke's Bay Farmland, New Zealand. J. Zool. 17: 243-255.

Lawlor, T.E., D.J. Hafner, P.T. Stapp, B.R. Riddle \& S.T. Alvarez-Castañeda. 2002. The Mammals. Pp.326-361. In: Case, Cody \& Ezcurra (eds.). A New Isaland Biogeography of the Sea Cortes. Universty of California Press.

León, J.L. \& R. Coria. 1992. Flora Iconográfica de Baja California Sur. Publicación No. 3. Centro de Investigaciones Biológicas de Baja California Sur. México. 156 pp.

León de la Luz, J.L., R. Coria-Benet, \& M. Cruz Estrada. 1996. Fenología de una comunidad Arido-Tropical de Baja California Sur, México. Acta Botánica Mexicana vol??

López-Forment, C., Lira, \& I., Müdespacher. 1996. Mamíferos de las Islas Mexicanas. AGT editor S.A. México pp. $25-27$

Mahlow, J. \& M. Slater. 1996. Current issues in the control of stray and feral cats. JAVMA, 209(12): 216-217.

Millar, J. 1988. Reproduction and survival of Peromyscus in seasonal environments. Special publication Carnegie Museum of Natural History. Pp.253-266.

Miller, C.J. \& T.K. Miller. 1995. Population dynamics and diet of rodents on Rangitoto island, New Zealand, including the effect of a 1080 Poison Operation. New Zealand J. Ecol. 19(1): 19-27.

Moors, P. \& E. Atkinson. 1984. Predation on Seabirds by introduced animals, and factors affecting its severity. ICBP Technical Publication No. 2: 667-690.

Murphy R.W. \& G. Aguirre-León. 2002. Non avian Reptiles: Origins and Evolution. 181-220 pp. In: Case, Cody \& Ezcurra (eds.). A New Island Biogeography of the Sea Cortes. Universty of California Press.

Nogales, M. \& F.M. Medina. 1996. A review of the diet of feral domestic cats (Felis silvestris f. Catus) on the Canary Islands, with new data from the laurel forest of La Gomera. Zeischrift fur Saugetierkunde 61:1-6.

Nogales, M., A. Martín, B. R. Tershy, C.J. Donland, D. Veitch, N. Puerta, B. Word \& J. Alonso. 2004. A review of feral cat eradications on islands. Conservation Biology, 18(2):310-319.

Rauzon, J. 1985. Feral cats on Jarvis island: Their effects and their eradication. Atoll Research Bulletin. 282.

Richman, A., T. Case, \& T. Schwaner. 1988. Natural and unnatural extinction rates of reptiles on islands. American Naturalist. 131(5):611-630.

Rodríguez, R., G. Arnaud., S. Alvarez. \& A. Rodríguez. 1991. Predation by feral cats on birds at isla Socorro, México. Western Birds 22:141-143.

Rodríguez-Moreno, A. 1997. Diagnostico de las islas del Golfo de California adyacentes a Baja California Sur, en relación con la presencia de gato doméstico (Felis catus). Tesis Profesional. Universidad Autónoma Metropolitana Unidad-Xochimilco.73 pp. 
Schofield, E. 1989. Effects of introduced Plants and Animals on Island vegetation: Examples from the Galápagos Archipelago. Biol.Conserv. 3(3):227-237.

Seabrook, W. 1990. The impact of the feral cat (Felis catus) on native fauna of Aldabra Atoll, Seychelles. Rev.Ecol. (Terre Vie),45:976 -985.

Smith, A.P. \& D.G. Quin. 1996. Patterns and causes of extinction and decline in Australian conilurine rodents. Biological Conservation 77:243-267.

Smith, F.A., B.T., Bestelmeyer., J., Biardi. \& M., Strong. 1993. Antropogenic extinction of the endemic woodrat, Neotoma bunkeri Burt. Biodiversity Letters 1:149-155.

Sokal, R.R. \& F.J. Rohlf. 1981. Biometry. W.H. Freeman and Company. New York U.S.A. 887 pp.

Tidemann, C.R., H.D., Yorkston \& A.J. Russack. 1994. The diet of cats, Felis catus, on Cristmas Island, Indian Ocean. Wildl. Res., 21:279-286.

Towns, D.R. 1991. Response of lizard assemblages in the Mercury Islands, New Zealand, to removal of an introduced rodent: the kiore (Rattus exulans). J. Roy. Soc.New Zeal. 21:119136.

Trujano, A. L. 2000. Estudio del ámbito hogareño de la comunidad de heterómidos del matorral sarcocaule en La Paz, Baja California Sur, México. Universidad Nacional Autónoma de México. Tesis de Licenciatura. 49 pp.

Van Aarde, J. 1983. Demographic parameters of feral cat Felis catus population at Marion Island: Selectives adventages. S. Afr. J. Wildl. Res., 13(1):12-16.

1984. Population biology and the control of feral cats on Marion Island. Acta Zool. Fennica 172: 107-110.

1986. A case study of an alien predator (Felis catus) introduced on Marion Island: Selectives adventages. S. Afr. J. Antarct. Res. 16(3):113-114.

Veitch, C. 1980. Feral cats on Little Barrier Island. Wildlife a Review No.11: 62-64. New Zealand Wildlife Service Departament of Internal Affairs.

1983. A cat problem removed. Wildlife a review No.12. pp.47-49. New Zealand Wildlife Service Departament of internal affairs.

1985. Methods of eradicating feral cats from offshore islands in New Zealand. ICBP Technical Publication 3: 271pp.

1989. The eradication of cat and sheep from Socorro Island. Northern Region Technical Report Series 11

Veitch, C., \& B. Bell. 1990. Eradication of introduced animals from the islands of New Zealand. Ecological Restauration of New Zealand Islands. Conservation Sciencies Publication.2.

White, G., D. Anderson, P. Burnham, \& D. Otis. 1982. Capture - Recapture and Removal Methods for Sampling Closed Populations. Los Alamos National Laboratory New Mexico. U.S.A. $x v i+235 \mathrm{pp}$.

W. W. F. 1980. Manual de Técnicas de Gestión de Vida Silvestre. Cuarta edición. 703 pp. 
